\title{
Analytic tools for IR subtraction beyond NLO
}

\author{
Lorenzo Magnea*, Ezio Maina, Giovanni Pelliccioli, Chiara Signorile-Signorile, \\ Paolo Torrielli and Sandro Uccirati \\ University of Torino and INFN, Sezione di Torino \\ E-mail: lorenzo.magnea@unito.it
}

\begin{abstract}
We review some of our recent work on the infrared subtraction problem beyond NLO, focusing in particular on the general definition of local subtraction counterterms. Our approach takes as a starting point the infrared factorisation of virtual corrections to fixed-angle scattering amplitudes in massless gauge theories, and uses that structure to identify a set of matrix elements of field operators and Wilson lines, which can be used as local soft and collinear counterterms to all orders in perturbation theory. Tracing the connection between factorisation and subtraction uncovers significant simplifications in the structure of counterterms, especially for nested singular regions, and we believe that it will be a crucial ingredient for the construction of minimal, stable, and efficient subtraction algorithms, at NNLO and beyond.
\end{abstract}

Loops and Legs in Quantum Field Theory (LL2018)

29 April 2018 - 04 May 2018

St. Goar, Germany

${ }^{*}$ Speaker 


\section{Introduction}

The problem of local subtraction of singular regions of integration for real radiation contributions to strong interaction cross sections has been the focus of intense research activity in recent years: the reason lies in the increasing precision requirements of LHC measurements, as integrated luminosity continues to grow, but also in the complexity of typical collider observables, which almost always requires a numerical treatment of radiative corrections, preventing an easy cancellation of infrared poles. The current precision standard is the Next-to-Next-to-Leading order (NNLO) in perturbation theory, but work towards extensions to the next order is already in progress. At NNLO, the case of event shapes and jet cross sections in $e^{+} e^{-}$annihilation was successfully tackled about a decade ago [1,2]; fully hadronic processes, however, have proved to be more challenging, and are currently being studied with a range of different methods. The Stripper framework [3] yielded the first fully differential results for hadronic processes with colourful final states, with the study of top quark pair production in $[4,5]$. More recently, a precise approximation to differential distributions for two-jet production was computed within the Antenna subtraction framework [6,7], which was further applied to jet production in Deep Inelastic Scattering [8] and to the associated production of a $W$ boson and a jet [9]. LHC processes with up to one final-state coloured particle at Born level have been analysed also using 'phase-space slicing' methods, such as $q_{T}$ slicing $[10,11,12]$ and $N$-Jettiness slicing $[13,14,15,16]$. A range of new methods are being developed and applied to selected processes: Projection-to-Born [17], the CoLoRFulNNLO framework [18, 19, 20], the technique of Nested Soft-Collinear subtractions [21, 22], the Unsubtraction [23] and the Geometric Subtraction ideas [24]. Finally, early studies of simple $\mathrm{N}^{3} \mathrm{LO}$ processes have recently been presented [25, 26, 27].

The NNLO techniques developed and implemented so far are very demanding, either from a numerical point of view, or because the required analytical integrations are very intricate. It is therefore not surprising that a number of groups are searching for ways to optimise and streamline existing algorithms: in particular, this is likely to be an essential requirement for the deployment of the methods in multi-particle configurations at NNLO, and in any case at $\mathrm{N}^{3} \mathrm{LO}$. In the present contribution we discuss our recent work [28, 29, 30, 31], where we presented a proof of principle for what we believe to be a highly efficient analytical subtraction scheme, and we studied the connection between the structure of local infrared counterterms for real radiation and the factorised structure of fixed-angle massless gauge-theory amplitudes. This second line of investigation, in particular, pursues two different but related goals. On the one hand, as we will briefly review here, it provides a list of gauge-invariant matrix elements of field operators and Wilson lines, which yield infrared local counterterms to any order in perturbation theory; in principle, this allows a direct calculation of counterterms without reference to any specific scattering amplitude, in contrast to the methods followed so far at the two-loop level $[32,33]$. On the other hand, tracing the connection between virtual corrections and real radiation, one may hope that the structural simplifications displayed by virtual factorisation will be reflected in a simplification of the counterterms. Indeed, we note that the infrared structure of fixed-angle scattering amplitudes is very well understood [34, 35, 36, 37, 38, 39, 40, 41, 42]: infrared singularities associated with on-shell configurations of loop momenta are known to factorise from the hard scattering; nested singular configurations (affected by both soft and collinear singularities) have a remarkably simple structure; finally, the factorised 
functions describing the singularities obey a pattern of exponentiation dictated by a small set of infrared anomalous dimensions, which (in the massless case) are known to three loops [43, 44]. For real radiation, existing knowledge is somewhat less developed: the factorisation properties of real radiation amplitudes, in the limits when one or more external partons become soft, or collinear to each other, are understood in considerable generality [45, 46, 47], and all the splitting kernels (at the amplitude level) necessary for next-to-next-to-leading order (NNLO) calculations have been computed [47, 48, 49, 50]; on the other hand, a complete all-order understanding of factorisation in all relevant limits is still lacking, in particular concerning the organisation of nested singular regions.

In the following, we will present some recent progress in these directions, focusing in particular on the derivation of general expressions for local infrared counterterms from the structure of virtual corrections. In Section 2, we will very briefly review the structure of infrared factorisation of fixed-angle scattering amplitudes; then, in Section 3, we will present a basic outline of the subtraction problem at NLO and NNLO, following the approach of Ref. [28]; in Section 4, we will present and motivate our general prescription to define local infrared subtraction counterterms to all orders in perturbation theory; in Section 5, we will give a few examples, at the NNLO level, showing how our procedure advantageously organises the subtraction counterterms, with an automatic treatment of nested singular regions; finally, we conclude with a brief perspective in Section 6 .

\section{Factorisation}

Given a scattering process with $n$ external partons, in massless perturbative QCD the IR behaviour of the corresponding amplitude is described by the factorisation formula

$$
\mathscr{A}_{n}\left(\frac{p_{i}}{\mu}\right)=\prod_{i=1}^{n}\left[\frac{\mathscr{J}_{i}\left(\left(p_{i} \cdot n_{i}\right)^{2} /\left(n_{i}^{2} \mu^{2}\right)\right)}{\mathscr{J}_{E, i}\left(\left(\beta_{i} \cdot n_{i}\right)^{2} / n_{i}^{2}\right)}\right] \mathscr{S}_{n}\left(\beta_{i} \cdot \beta_{j}\right) \mathscr{H}_{n}\left(\frac{p_{i} \cdot p_{j}}{\mu^{2}}, \frac{\left(p_{i} \cdot n_{i}\right)^{2}}{n_{i}^{2} \mu^{2}}\right),
$$

where the dependence on the renormalised coupling $\alpha_{\mathrm{s}}(\mu)$ and on the dimensional regulator $\varepsilon=$ $2-d / 2$ has been suppressed to simplify the notation. Color indices have also been suppressed, but it should be kept in mind that $\mathscr{A}_{n}$ is a vector in the space of color configurations for the amplitude, while the soft function $\mathscr{S}_{n}$, generating all soft poles, is a color operator in the same space, acting on the finite hard vector remainder $\mathscr{H}_{n}$. The jet functions $\mathscr{J}_{i}$ and $\mathscr{J}_{E, i}$, on the other hand, are colour-singlet quantities assigned to individual hard partons. The factorisation of the amplitude in Eq. (2.1) expresses the universal structure of infrared singularities, contained in the soft and collinear functions, $\mathscr{S}_{n}$ and $\mathscr{J}_{i}$, respectively, so that the only process-dependent part is the hard reminder $\mathscr{H}_{n}$, which is finite in $d=4$ after renormalisation. $\mathscr{H}_{n}$ is defined by matching, in such a way that the unphysical dependence on the auxiliary factorisation vectors $n_{i}^{\mu}, n_{i}^{2} \neq 0$ cancels in the full amplitude.

The functions entering Eq. (2.1) can be explicitly defined in terms of matrix elements of field operators and Wilson lines. To begin with, to collect all the collinear singularities of the process we introduce for each external hard parton $i$ the corresponding jet function $\mathscr{J}_{i}\left(p_{i}, n_{i} ; \mu\right)$. For example, 
for an outgoing quark, the explicit expression is

$$
\bar{u}_{s}(p) \mathscr{J}_{q}\left(\frac{(p \cdot n)^{2}}{n^{2} \mu^{2}}\right)=\left\langle p, s\left|\bar{\psi}(0) \Phi_{n}(0, \infty)\right| 0\right\rangle,
$$

where the Wilson line operator is the path ordered gauge phase

$$
\Phi_{n}\left(\lambda_{2}, \lambda_{1}\right) \equiv \mathscr{P} \exp \left\{\mathrm{i} g \int_{\lambda_{1}}^{\lambda_{2}} d \lambda v \cdot A(\lambda v)\right\}
$$

As a consequence of gauge invariance and diagrammatic power counting, the collinear functions $\mathscr{J}_{i}$ do not involve color correlation. By contrast, as announced above, the soft function $\mathscr{S}_{n}$ is a color operator mixing the available color tensors, and it is defined as a correlator of light-like Wilson lines running along the classical trajectories of the hard partons. One writes

$$
\mathscr{S}_{n}\left(\beta_{i} \cdot \beta_{j}\right)=\left\langle 0\left|\prod_{k=1}^{n} \Phi_{\beta_{k}}(\infty, 0)\right| 0\right\rangle
$$

Finally, the denominator in the factorisation formula is the product of eikonal jet functions, one for each hard parton: their introduction avoids the double counting of soft-collinear singularities, which are present both in the numerator jets and in the soft operator. Eikonal jets are soft approximations to ordinary jets, and they can be defined replacing field operators with Wilson lines in the jet definition. One finds then

$$
\mathscr{J}_{E}\left(\frac{\left(\beta_{i} \cdot n\right)^{2}}{n^{2}}\right)=\left\langle 0\left|\Phi_{\beta}(\infty, 0) \Phi_{n}(\infty, 0)\right| 0\right\rangle
$$

where the Wilson lines must be taken in the color representation of the original hard parton. The ratio of the jet function $\mathscr{J}_{i}\left(p_{i}, n ; \mu\right)$ with its eikonal counterpart contains only hard-collinear poles, a fact which has significant implications for subtraction in the case of nested singular regions, as we will briefly discuss below.

\section{Subtraction}

We start our discussion sketching the general structure of subtraction at NLO and NNLO in massless QCD. For simplicity, we will focus on processes with coloured particles appearing only in the final state, a typical example being $e^{+} e^{-} \rightarrow$ jets. Consider then a process which at LO involves $n$ final state partons. Given an infrared-safe observable $X$, the NLO correction to the distribution of $X$ can be expressed as

$$
\frac{d \sigma_{\mathrm{NLO}}}{d X}=\left(\int d \Phi_{n} V_{n} \delta_{n}(X)+\int d \Phi_{n+1} R_{n+1} \delta_{n+1}(X)\right)_{d=4},
$$

where $V_{n}$ and $R_{n+1}$ account respectively for one-loop virtual and single-real radiation corrections to the Born squared matrix element $B_{n} . \delta_{n}(X)$ and $\delta_{n+1}(X)$ localise the integrals to the chosen value of the observable $X$, evaluated respectively with $n$-particle and $(n+1)$-particle kinematics. The fact that $X$ is an infrared-safe observable implies that $X_{n+1} \rightarrow X_{n}$ in the limit of one-unresolved (either soft or collinear) parton. Thanks to the KLN theorem, the explicit IR poles of $V_{n}$ are then exactly 
cancelled by the poles which arise from the integration of $R_{n+1}$ over the radiation phase-space: this makes the final answer finite in $d=4$.

The computation of the r.h.s. of Eq. 3.1 is in general hard, since both terms must be computed in $d=4-2 \varepsilon$, and due to the complexity of the typical observable $X$, and of the amplitudes. The subtraction procedure improves the situation by allowing for numerical calculations entirely performed in $d=4$. To this end, one builds a set of local counterterms $K_{i}$, incorporating the same singular behaviour of $R_{n+1}$ in the unresolved limits, and at the same time simple enough to be analytically integrated over the radiation phase-space. Thanks to the factorization of the $(n+1)$ particle phase-space measure, we can write the counterterm contribution to the observable $X$ as

$$
\left.\frac{d \sigma_{\mathrm{NLO}}}{d X}\right|_{\mathrm{ct}}=\int d \Phi_{n+1} K_{n+1} \delta_{n}(X)=\int d \Phi_{n} d \Phi_{1} K_{n+1} \delta_{n}(X)
$$

Defining then the integrated counterterm

$$
I_{n}=\int d \Phi_{1} K_{n+1}
$$

one can identically recast Eq. (3.1) in subtracted form as

$$
\begin{aligned}
\left.\frac{d \sigma_{\mathrm{NLO}}}{d X}\right|_{\text {subtr }}=\frac{d \sigma_{\mathrm{NLO}}}{d X}= & \int d \Phi_{n}\left[V_{n}+I_{n}\right]_{d=4} \delta_{n}(X) \\
& +\int\left[d \Phi_{n+1} R_{n+1} \delta_{n+1}(X)-d \Phi_{n+1} K_{n+1} \delta_{n}(X)\right]_{d=4} .
\end{aligned}
$$

Note that in this form the integrands of the first and the second line are separately finite and integrable in four dimensions, thus they can be integrated numerically.

At NNLO, the logic of subtraction is the same, but, since the pattern of cancellation of IR singularities among real and virtual contributions is much more involved, the procedure is technically more complicated. In the same fashion of Eq. (3.1), we can write the NNLO correction to the distribution of $X$ as

$$
\frac{d \sigma_{\mathrm{NNLO}}}{d X}=\left(\int d \Phi_{n} V V_{n} \delta_{n}(X)+\int d \Phi_{n+1} R V_{n+1} \delta_{n+1}(X)+\int d \Phi_{n+2} R R_{n+2} \delta_{n+2}(x)\right)_{d=4} .
$$

At this level, IR poles arise from three different regimes: the double-real contribution $R R_{n+2}$ features configurations in which either one or two radiated particles become unresolved; furthermore, the real-virtual $R V_{n+1}$ features configurations with one radiation going unresolved. This suggests that three local counterterms functions have to be constructed: we denote by $K_{n+2}$ a function incorporating the same phase-space singularities as $R R_{n+2}$ when both radiated particles go unresolved, while $K_{n+2}^{(\mathbf{1})}$ has the same singular behaviour of $R R_{n+2}$ when only one radiated parton goes unresolved; finally, $K_{n+1}^{(\mathbf{R V})}$ approximates the IR divergences of the real-virtual squared amplitude $R V_{n+1}$, when the single radiated particle becomes unresolved. The expressions for the integrated counterterms to be summed and subtracted in Eq. 3.5 are then

$$
\int d \Phi_{n+2} K_{n+2} \delta_{n}(X), \quad \int d \Phi_{n+2} K_{n+2}^{(\mathbf{1})} \delta_{n+1}(X), \quad \int d \Phi_{n+1} K_{n+1}^{(\mathbf{R V})} \delta_{n}(X) .
$$

As discussed in Ref. [28], it turns out to be convenient to separate the double-unresolved counterterm $K_{n+2}$, in order to distinguish between hierarchic double-unresolved singular behaviours, where 
one of the two particles becomes unresolved at a faster rate, and other configurations where both particles become unresolved at the same rate. We write then

$$
K_{n+2}=K_{n+2}^{(\mathbf{1 2})}+K_{n+2}^{(\mathbf{2 2})} .
$$

We now have four integrated counterterms, which take the form

$$
\begin{aligned}
I_{n+1}^{(\mathbf{1 2})}=\int d \Phi_{1} K_{n+2}^{(\mathbf{1 2})}, & I_{n}^{(\mathbf{2 2})}=\int d \Phi_{2} K_{n+2}^{(\mathbf{2 2})} \\
I_{n+1}^{(\mathbf{1})}=\int d \Phi_{1} K_{n+2}^{(\mathbf{1})}, & I_{n}^{(\mathbf{R V})}=\int d \Phi_{1} K_{n+1}^{(\mathbf{R V})} .
\end{aligned}
$$

Using these definitions, we can now rewrite identically the NNLO corrections to the distribution of $X$, Eq. (3.5), in subtracted form, as

$$
\begin{aligned}
\left.\frac{d \sigma_{\mathrm{NNLO}}}{d X}\right|_{\text {subtr }} & =\int d \Phi_{n}\left[V V_{n}+I_{n}^{(\mathbf{2 2})}+I_{n}^{(\mathbf{R V})}\right]_{d=4} \delta_{n}(X) \\
& +\int d \Phi_{n+1}\left[\left(R V_{n+1}+I_{n+1}^{(\mathbf{1})}\right) \delta_{n+1}(X)-\left(K_{n+1}^{(\mathbf{R V})}-I_{n+1}^{(\mathbf{1 2})}\right) \delta_{n}(X)\right]_{d=4} \\
& +\int d \Phi_{n+2}\left[R R_{n+2} \delta_{n+2}(X)-K_{n+2}^{(\mathbf{1})} \delta_{n+1}(X)-\left(K_{n+2}^{(\mathbf{1 2})}+K_{n+2}^{(\mathbf{2 2})}\right) \delta_{n}(X)\right]_{d=4} .
\end{aligned}
$$

The first line is characterised by a Born-like kinematics, and is finite in $d=4$, as the sum $I_{n}^{(\mathbf{2 2})}+$ $I_{n}^{(\mathbf{R V})}$ contains explicit poles in $\varepsilon$ which exactly cancel those of $V V_{n}$. Regarding the second line, the poles in $I_{n+1}^{(\mathbf{1})}$ cancel those of $R V_{n+1}$ : their sum is finite in four dimensions, but still features single-radiation phase-space singularities, which should be cancelled. $K_{n+1}^{(\mathbf{R V})}$ cancels the phasespace singularities of $R V_{n+1}$ but features explicit poles, which are cancelled by $I_{n+1}^{(\mathbf{1 2})}$. Further, the phase space singularities of $K_{n+1}^{(\mathbf{R V})}-I_{n+1}^{(\mathbf{1 2})}$ exactly cancel those of $I_{n+1}^{(\mathbf{1})}+R V_{n+1}$ : the full second line is then finite and integrable in the single-radiation phase space. Finally, the three integrands which appear in the third line of Eq. (3.9) are separately finite in $d=4$ and their sum is integrable in $d \Phi_{\mathrm{rad}, 2}$, thus suitable for numerical evaluation.

It is clear the above outline is just a sketch of a complete subtraction procedure: in order to have a working algorithm, one must identify counterterm functions that are simple enough to be integrated analytically, one must construct explicit phase space mappings in order to work at all time with on-shell momentum-conserving configurations, and one must have a complete procedure to subtract double-counted nested singular regions. Our approach to these problems is described in Ref. [28]. In what follows, we will focus instead on a general procedure to construct subtraction counterterms, to any order in perturbation theory, which arises out of the factorisation described in Section 2: the simple treatment of soft-collinear regions in the virtual case will be reflected in the structure of the real-radiation counterterms.

\section{Local Counterterms}

We now illustrate how infrared factorisation of fixed-angle scattering amplitudes can be used to infer general definitions for soft and collinear local counterterms to all orders. 
Let us begin with soft radiation. For a generic process involving the emission of $m$ soft partons from $n$ hard ones, we argue that the soft singularities of the real-radiation amplitude are reproduced by eikonal form factors, defined by

$$
\begin{aligned}
\mathscr{S}_{n, m}\left(k_{1}, \ldots, k_{m} ; \beta_{i}\right) & \equiv\left\langle k_{1}, \lambda_{1} ; \ldots ; k_{m}, \lambda_{m}\left|\prod_{i=1}^{n} \Phi_{\beta_{i}}(\infty, 0)\right| 0\right\rangle \\
& \equiv \varepsilon_{\mu_{1}}^{*\left(\lambda_{1}\right)}\left(k_{1}\right) \ldots \varepsilon_{\mu_{m}}^{*\left(\lambda_{m}\right)}\left(k_{m}\right) J_{\mathscr{S}}^{\mu_{1} \ldots \mu_{m}}\left(k_{1}, \ldots, k_{m} ; \beta_{i}\right) \\
& \equiv \sum_{p=0}^{\infty} \mathscr{S}_{n, m}^{(p)}\left(k_{1}, \ldots, k_{m} ; \beta_{i}\right),
\end{aligned}
$$

where in the second line we define multiple soft-gluon emission currents, and the third line defines the perturbative expansion of the form factor, which includes loop corrections at all orders in perturbation theory. Neglecting for the moment collinear singularities, the underlying assumption is that the real radiation matrix element factorises, at leading power in the soft momenta, as

$$
\mathscr{A}_{n, m}\left(k_{1}, \ldots, k_{m} ; p_{i}\right)=\mathscr{S}_{n, m}\left(k_{1}, \ldots, k_{m} ; \beta_{i}\right) \mathscr{H}_{n}\left(p_{i}\right)+\mathscr{R}_{n, m},
$$

with corrections that are finite in dimensional regularisation and integrable in the soft-gluon phase space. At cross section level, one then writes

$$
\sum_{\lambda_{i}}\left|\mathscr{A}_{n, m}\left(k_{1}, \ldots, k_{m} ; p_{i}\right)\right|^{2} \simeq \mathscr{H}_{n}^{\dagger}\left(p_{i}\right) S_{n, m}\left(k_{1}, \ldots, k_{m} ; \beta_{i}\right) \mathscr{H}_{n}\left(p_{i}\right),
$$

where the radiative soft function $S_{n, m}$ is defined as a Wilson-line squared matrix elements for fixed final state quantum numbers, writing

$$
\begin{aligned}
S_{n, m}\left(k_{1}, \ldots, k_{m} ; \beta_{i}\right) & \equiv \sum_{p=0}^{\infty} S_{n, m}^{(p)}\left(k_{1}, \ldots, k_{m} ; \beta_{i}\right) \\
& \equiv \sum_{\lambda_{i}}\left\langle 0\left|\prod_{i=1}^{n} \Phi_{\beta_{i}}(0, \infty)\right| k_{1}, \lambda_{1} ; \ldots ; k_{m}, \lambda_{m}\right\rangle\left\langle k_{1}, \lambda_{1} ; \ldots ; k_{m}, \lambda_{m}\left|\prod_{i=1}^{n} \Phi_{\beta_{i}}(\infty, 0)\right| 0\right\rangle .
\end{aligned}
$$

Summing over soft particle numbers and integrating over the soft phase space one can build a fully inclusive eikonal cross section, which is finite order by order in perturbation theory by general theorems, and, by completeness, can be written as

$$
\sum_{m=0}^{\infty} \int d \Phi_{m} S_{n, m}\left(k_{1}, \ldots, k_{m} ; \beta_{i}\right)=\left\langle 0\left|\prod_{i=1}^{n} \Phi_{\beta_{i}}(0, \infty) \prod_{i=1}^{n} \Phi_{\beta_{i}}(\infty, 0)\right| 0\right\rangle .
$$

The finiteness of Eq. (4.5) shows that the eikonal form factors defined in Eq. (4.1) are well-suited to provide local soft counterterms for the emission of $m$ unresolved gluons, order by order in the strong coupling.

A similar reasoning, starting with the operator expressions for virtual jets and eikonal jets, yields the definition of collinear and soft-collinear local counterterms. For example, for a single emitting quark with momentum $p$ and spin $s$, amplitude-level radiative jet functions for the emission of $m$ collinear gluons can be defined as

$$
\begin{aligned}
\bar{u}_{S}(p) \mathscr{J}_{q, m}\left(k_{1}, \ldots, k_{m} ; p, n\right) & \equiv\left\langle p, s ; k_{1}, \lambda_{1} ; \ldots ; k_{m}, \lambda_{m}\left|\bar{\psi}(0) \Phi_{n}(0, \infty)\right| 0\right\rangle \\
& \equiv \bar{u}_{s}(p) \sum_{p=0}^{\infty} \mathscr{J}_{q, m}^{(p)}\left(k_{1}, \ldots, k_{m} ; p, n\right)
\end{aligned}
$$


where for $m=0$ we recover the virtual jet function of Eq. (2.2). Unlike the soft function, the jet does not depend on the quantum numbers of other partons in the scattering amplitude; on the other hand, it depends on the spin of the emitter, whereas eikonal functions are only sensitive to colors and directions of the hard partons. At cross-section level, the radiative jet function, involves a Fourier transform, necessary to encode the non-trivial momentum flow. We write

$$
\begin{aligned}
J_{q, m}\left(k_{1}, \ldots k_{m} ; l, p, n\right) & \equiv \sum_{p=0}^{\infty} J_{q, m}^{(p)}\left(k_{1}, \ldots k_{m} ; l, p, n\right) \\
& \equiv \int d^{d} x \mathrm{e}^{\mathrm{i} l \cdot x} \sum_{\left\{\lambda_{j}\right\}}\left\langle 0\left|\Phi_{n}(\infty, x) \psi(x)\right| p, s ; k_{j}, \lambda_{j}\right\rangle\left\langle p, s ; k_{j}, \lambda_{j}\left|\bar{\psi}(0) \Phi_{n}(0, \infty)\right| 0\right\rangle .
\end{aligned}
$$

As was done for the radiative soft functions, also for jets we can sum over final states, and use completeness to construct a fully inclusive, and thus finite, collinear cross-section, given by

$$
\begin{aligned}
& \sum_{m=0}^{\infty} \int d \Phi_{m+1} J_{q, m}\left(k_{1}, \ldots, k_{m} ; l, p, n\right)= \\
& \operatorname{Disc}\left[\int d^{d} x \mathrm{e}^{\mathrm{i} l \cdot x}\left\langle 0\left|\Phi_{n}(\infty, x) \psi(x) \bar{\psi}(0) \Phi_{n}(0, \infty)\right| 0\right\rangle\right] .
\end{aligned}
$$

The finiteness of Eq. (4.8) shows that the cross-section-level radiative jets defined in Eq. (4.8) are suitable as local collinear counterterms. Let us remark, however, that at this stage the collinear limit has still to be taken, unlike the soft case. Moreover, special attention is needed in the choice of the auxiliary Wilson-line vector $n^{\mu}$, which is a necessary ingredient for the gauge invariance of the jet function: a light-like $n^{\mu}$ simplifies considerably the analytic computation, but, at loop level, it has the price of introducing spurious collinear singularities, related to the emission from the auxiliary Wilson line.

The final element in our list of counterterms is a local function simulating the squared matrix element in the kinematic region where radiated particles are both soft and collinear to one of the hard partons. Such a function allows for the subtraction of the double counting of these regions, which are reproduced by both the soft operator and the selected jet function. To this end, we introduce the (cross-section-level) eikonal jet function

$$
\begin{aligned}
J_{E, m}\left(k_{j} ; l, \beta, n\right) & \equiv \sum_{p=0}^{\infty} J_{E, m}^{(p)}\left(k_{j} ; l, \beta, n\right) \\
& \equiv \int d^{d} x \mathrm{e}^{\mathrm{i} l \cdot x}\left\langle 0\left|\Phi_{n}(\infty, x) \Phi_{\beta}(x, \infty)\right| k_{j}, \lambda_{j}\right\rangle\left\langle k_{j}, \lambda_{j}\left|\Phi_{\beta}(\infty, 0) \Phi_{n}(0, \infty)\right| 0\right\rangle .
\end{aligned}
$$

As is the case for virtual corrections ( $m=0$ in Eq. (4.9)), subtracting the eikonal radiative jet from the ordinary jet returns a purely hard-collinear contribution associated with the selected hard parton.

As a simple proof of concept, we note here that the explicit calculation of $S, J$ and $J_{E}$ at the lowest non-trivial perturbative order (i.e. with the emission of a single gluon) reproduces the wellknown results for the tree-level eikonal current and the leading order DGLAP splitting kernels, respectively. For the soft function, in the simple case with only two Wilson lines, one easily finds

$$
S_{2,1}\left(k ; \beta_{i}\right)=\sum_{\lambda}\left\langle 0\left|\prod_{i=1}^{2} \Phi_{\beta_{i}}(0, \infty)\right| k, \lambda\right\rangle\left\langle k, \lambda\left|\prod_{i=1}^{2} \Phi_{\beta_{i}}(\infty, 0)\right| 0\right\rangle=-2 g^{2} \mathbf{T}_{1} \cdot \mathbf{T}_{2} \frac{\beta_{1} \cdot \beta_{2}}{\beta_{1} \cdot k \beta_{2} \cdot k},
$$


matching the well-known result for a single radiation. Similarly, the tree-level calculation of the radiative quark jet function $J_{q, 1}$ involves three diagrams that, summed over fermion spins and gluon polarisations, in Feynman gauge, yield

$$
\sum_{s} J_{q, 1}(k ; l, p, n)=\frac{4 \pi \alpha_{s} C_{F}}{l^{2}}(2 \pi)^{d} \delta^{d}(l-p-k)\left[-l \gamma_{\mu} \not p \gamma^{\mu} l+\frac{l^{2}}{k \cdot n}(l, h p p+\not p h l)\right],
$$

up to terms proportional to $n^{2}$. Then, choosing $l^{\mu}$ as the collinear direction and $l_{\perp}^{\mu}$ as a reference vector perpendicular to $p^{\mu}$ and $k^{\mu}$, we can set

$$
p^{\mu}=z l^{\mu}+\mathscr{O}\left(l_{\perp}\right), \quad k^{\mu}=(1-z) l^{\mu}+\mathscr{O}\left(l_{\perp}\right), \quad n^{2}=0 .
$$

We can now focus on the leading behaviour of $J_{q, 1}$ as $k_{\perp}^{\mu} \rightarrow 0$, and we find

$$
\sum_{s, \lambda} J_{q, 1}(k ; l, p, n)=\frac{8 \pi \alpha_{s} C_{F}}{l^{2}}(2 \pi)^{d} \delta^{d}(l-p-k)\left[\frac{1+z^{2}}{1-z}-\varepsilon(1-z)+\mathscr{O}\left(l_{\perp}\right)\right],
$$

reconstructing, as expected, the spin-averaged DGLAP splitting kernel for the branching $q \rightarrow q g$. Finally, we look at the soft-collinear local counterterm for single radiation, which gives

$$
\sum_{\lambda} J_{E, m}(k ; l, \beta, n)=g^{2}(2 \pi)^{d} \boldsymbol{\delta}(l-p) \frac{2 p \cdot n}{p \cdot k n \cdot k} .
$$

In the collinear limit (up to corrections of order $l_{\perp}$ ) we can set

$$
l^{2}=(p+k)^{2}=2 p \cdot k, \quad p \cdot n=z l \cdot n, \quad k \cdot n=(1-z) l \cdot n,
$$

so that the soft-collinear counterterm takes the form

$$
\sum_{\lambda} J_{E, m}(k ; l, \beta, n)=\frac{8 \pi \alpha_{s} C_{F}}{l^{2}}(2 \pi)^{d} \delta(l-p) \frac{2 z}{1-z} .
$$

Notice that the factor $2 z$ in the numerator is crucial in order to prove that soft and collinear limits commute (at least at NLO), which in turn simplifies considerably the structure of the subtraction algorithm [28].

\section{NNLO examples}

The structure of counterterms that can be derived from virtual factorisation at NLO is standard, and is reviewed in $[28,29,30,31]$. Here we will instead focus on the more interesting case of NNLO counterterms, where the intricacy of nested subtractions comes into play. Our first step is to expand the factorisation formula for the fixed-angle $n$-point amplitude, Eq. (2.1) to order $g_{s}^{4}$. We find

$$
\begin{aligned}
\mathscr{A}_{n}^{(2)}\left(p_{i}\right)= & \mathscr{S}_{n}^{(2)}\left(\beta_{i}\right) \mathscr{H}_{n}^{(0)}\left(p_{i}\right)+\mathscr{S}_{n}^{(0)}\left(\beta_{i}\right) \mathscr{H}_{n}^{(2)}\left(p_{i}\right)+\mathscr{S}_{n}^{(1)}\left(\beta_{i}\right) \mathscr{H}_{n}^{(1)}\left(p_{i}\right) \\
& +\sum_{i=1}^{n}\left[\mathscr{J}_{i}^{(2)}\left(p_{i}\right)-\mathscr{J}_{E, i}^{(2)}\left(\beta_{i}\right)-\mathscr{J}_{E, i}^{(1)}\left(\beta_{i}\right)\left(\mathscr{J}_{i}^{(1)}\left(p_{i}\right)-\mathscr{J}_{E, i}^{(1)}\left(\beta_{i}\right)\right)\right] \mathscr{A}_{n}^{(0)}\left(p_{i}\right) \\
& +\sum_{i, j>i}^{n}\left(\mathscr{J}_{i}^{(1)}\left(p_{i}\right)-\mathscr{J}_{E, i}^{(1)}\left(\beta_{i}\right)\right)\left(\mathscr{J}_{j}^{(1)}\left(p_{j}\right)-\mathscr{J}_{E, j}^{(1)}\left(\beta_{j}\right)\right) \mathscr{A}_{n}^{(0)}\left(p_{i}\right) \\
& +\sum_{i=1}^{n}\left(\mathscr{J}_{i}^{(1)}\left(p_{i}\right)-\mathscr{J}_{E, i}^{(1)}\left(\beta_{i}\right)\right)\left[\mathscr{S}_{n}^{(1)}\left(\beta_{i}\right) \mathscr{H}_{n}^{(0)}\left(p_{i}\right)+\mathscr{S}_{n}^{(0)}\left(\beta_{i}\right) \mathscr{H}_{n}^{(1)}\left(p_{i}\right)\right] .
\end{aligned}
$$


It is not difficult to indentify the physical origin of soft and collinear poles described in Eq. (5.1). A slightly more cumbersome task is to construct the double-virtual contribution to the squared matrix element $\left(V V_{n}\right.$ in Eq. (3.5)), which involves the product of Eq. (5.1) with the Born amplitude, as well as the square of the one-loop amplitude. Furthermore, one has to organise the result in terms of cross-section-level jet and soft virtual functions, in order to be able to use the completeness relations in Eq. (4.5) and Eq. (4.8) to provide appropriate real-radiation counterterms. We summarise the structure of poles in the NNLO virtual correction as

$$
\begin{aligned}
(V V)_{n} \equiv & (V V)_{n}^{(2 \mathrm{~s})}+(V V)_{n}^{(1 \mathrm{~s})}+\sum_{i=1}^{n}(V V)_{n, i}^{(1 \mathrm{hc}, 1 \mathrm{~s})} \\
& +\sum_{i=1}^{n}(V V)_{n, i}^{(2 \mathrm{hc})}+\sum_{i, j>i=1}^{n}(V V)_{n, i j}^{(2 \mathrm{hc})}+\sum_{i=1}^{n}(V V)_{n, i}^{(1 \mathrm{hc})}
\end{aligned}
$$

where (2s) and (1s) denote double soft and single soft contributions, respectively, in each case including also soft-collinear singularities; similarly, (1hc) and (2hc) identify single and double hard-collinear poles, respectively: the double hard-collinear component is split into two terms, according to whether one or two hard partons are involved in the collinear emission; finally, (1hc, 1s) denotes terms with a hard-collinear pole associated with direction $i$, accompanied by a soft pole.

To illustrate the next steps, consider the structure of the double collinear radiation along hard parton $i$, which is given by

$$
(V V)_{n, i}^{(2 \mathrm{hc})}=\left[J_{i, 0}^{(2)}-J_{E, i, 0}^{(2)}-J_{E, i, 0}^{(1)}\left(J_{i, 0}^{(1)}-J_{E, i, 0}^{(1)}\right)\right]\left|\mathscr{A}_{n}^{(0)}\right|^{2} .
$$

The completeness relation in Eq. (4.8) (and its eikonal counterpart) implies in particular

$$
\begin{aligned}
& {\left[J_{E, i, 0}^{(1)}+\int d \Phi_{1} J_{E, i, 1}^{(0)}\right]\left[J_{i, 0}^{(1)}-J_{E, i, 0}^{(1)}+\int d \Phi_{1}^{\prime}\left(J_{i, 1}^{(0)}-J_{E, i, 1}^{(0)}\right)\right]=\text { finite, }} \\
& J_{i, 0}^{(2)}+\int d \Phi_{1} J_{i, 1}^{(1)}+\int d \Phi_{2} J_{i, 2}^{(0)}=\text { finite }
\end{aligned}
$$

which suggest defining the hard collinear counterterms

$$
\begin{aligned}
K_{n+2, i}^{(\mathrm{hc})} & =\left[J_{i, 2}^{(0)}-J_{E, i, 2}^{(0)}-J_{E, i, 1}^{(0)}\left(J_{i, 1}^{(0)}-J_{E, i, 1}^{(0)}\right)\right]\left|\mathscr{A}_{n}^{(0)}\right|^{2}, \\
K_{n+1, i}^{(\mathbf{R V}, \mathrm{hc})} & =\left[J_{i, 1}^{(1)}-J_{E, i, 1}^{(1)}-J_{i, 0}^{(1)} J_{E, i, 1}^{(0)}-J_{E, i, 0}^{(1)} J_{i, 1}^{(0)}+2 J_{E, i, 0}^{(1)} J_{E, i, 1}^{(0)}\right]\left|\mathscr{A}_{n}^{(0)}\right|^{2} .
\end{aligned}
$$

The first line in Eq. (5.5) is assigned to the double-unresolved counterterm, since all terms involve two radiations and no loop corrections. By contrast, the terms in the second line involve just one radiation, and a loop correction: they are therefore properly assigned to the real-virtual counterterm. The single-unresolved hard-collinear counterterm can similarly be determined by considering the single-radiation contributions to the $(n+1)$-particle matrix element.

As a second example, consider the single and the double soft contributions to the virtual correction. They can be written as

$$
\begin{aligned}
& (V V)_{n}^{(2 \mathrm{~s})}=\mathscr{H}_{n}^{(0) \dagger} S_{n, 0}^{(2)} \mathscr{H}_{n}^{(0)}, \\
& (V V)_{n}^{(1 \mathrm{~s})}=\mathscr{H}_{n}^{(0) \dagger} S_{n, 0}^{(1)} \mathscr{H}_{n}^{(1)}+\mathscr{H}_{n}^{(1) \dagger} S_{n, 0}^{(1)} \mathscr{H}_{n}^{(0)} .
\end{aligned}
$$


We are led to focus on the NLO and NNLO completeness relation derived from Eq. (4.5). They imply

$$
\begin{aligned}
& S_{n, 0}^{(1)}\left(\beta_{i}\right)+\int d \Phi_{1} S_{n, 1}^{(0)}\left(k, \beta_{i}\right)=\text { finite } \\
& S_{n, 0}^{(2)}\left(\beta_{i}\right)+\int d \Phi_{1} S_{n, 1}^{(1)}\left(k, \beta_{i}\right)+\int d \Phi_{2} S_{n, 2}^{(0)}\left(k_{1}, k_{2}, \beta_{i}\right)=\text { finite. }
\end{aligned}
$$

As before, we can assign terms with two radiations to the double-unresolved counterterm, here for the soft sector, while terms with a single radiation and a loop correction yield the real virtual soft counterterm. We get

$$
\begin{aligned}
K_{n+2}^{(\mathrm{s})} & =\mathscr{H}_{n}^{(0) \dagger} S_{n, 2}^{(0)} \mathscr{H}_{n}^{(0)}, \\
K_{n+1}^{(\mathbf{R V}, \mathrm{s})} & =\mathscr{H}_{n}^{(0) \dagger} S_{n, 1}^{(0)} \mathscr{H}_{n}^{(1)}+\mathscr{H}_{n}^{(1) \dagger} S_{n, 1}^{(0)} \mathscr{H}_{n}^{(0)}+\mathscr{H}_{n}^{(0) \dagger} S_{n, 1}^{(1)} \mathscr{H}_{n}^{(0)} .
\end{aligned}
$$

For completeness, we give also the expressions for the single-unresolved soft and hard-collinear counterterms, which have $(n+1)$-particle kinematics. They are given by

$$
\begin{aligned}
& K_{n+2}^{(\mathbf{1}, \mathrm{s})}=\mathscr{H}_{n+1}^{(0) \dagger} S_{n+1,1}^{(0)} \mathscr{H}_{n+1}^{(0)}, \\
& K_{n+2, i}^{(\mathbf{1}, \mathrm{hc})}=\left(J_{i, 1}^{(0)}-J_{E, i, 1}^{(0)}\right)\left|\mathscr{A}_{n+1}^{(0)}\right|^{2} .
\end{aligned}
$$

Upon integration over the appropriate radiation phase spaces, the listed local counterterms will generate soft and collinear single and double poles: by Eq. (4.5) and Eq. (4.8), these poles will cancel the known virtual singularities, leaving behind a finite and computable remainder. We note that the double counting of soft-collinear regions has naturally been taken care of, with soft-collinear poles assigned to the soft counterterms, while collinear counterterms contain exclusively hard contributions.

\section{Perspective}

We have given a brief account of some aspects of our approach to the IR subtraction problem beyond NLO. Specifically, we have presented evidence that the factorisation of virtual corrections to fixed-angle multi-particle massless gauge theory amplitudes contains important information for the structure of local subtraction counterterms. Operator expressions generating soft, collinear and soft-collinear poles at amplitude level suggest the definitions of eikonal form factors and radiative jet functions, encoding the singular behaviour of real radiation at all loop orders. These functions and form factors, upon integration over the radiation phase space, must cancel the virtual poles, as can be seen by simple completeness relations and the application of general cancellation theorems. The simple organisation of soft and collinear singularities in virtual corrections then reflects into the structure of real-radiation counterterms. In a separate publication [28] we have described in detail the other technical tools that are required to turn these considerations into a full-fledged subtraction algorithm: the organisation of the radiation phase space in sectors with appropriate sector functions, the phase space mappings allowing the use of on-shell scattering amplitudes for all multiplicities, and the necessary integrations for local counterterms. We hope that our approach will prove useful in the streamlining and optimisation of existing NNLO subtraction techniques, 
especially in view of multi-particle applications; we also believe that the information extracted from virtual factorisation will be crucial for the feasibility of general and efficient subtraction algorithm at $\mathrm{N}^{3} \mathrm{LO}$, when all the relevant tools become available. Work in these directions is in progress.

\section{References}

[1] A. Gehrmann-De Ridder, T. Gehrmann, E. W. N. Glover and G. Heinrich, Phys. Rev. Lett. 100 (2008) 172001 [arXiv:0802.0813 [hep-ph]].

[2] S. Weinzierl, Phys. Rev. Lett. 101 (2008) 162001 [arXiv:0807.3241 [hep-ph]].

[3] M. Czakon, Phys. Lett. B 693 (2010) 259 doi:10.1016/j.physletb.2010.08.036 [arXiv:1005.0274 [hep-ph]].

[4] M. Czakon, D. Heymes and A. Mitov, Phys. Rev. Lett. 116 (2016) no.8, 082003 [arXiv:1511.00549 [hep-ph]].

[5] M. Czakon, P. Fiedler, D. Heymes and A. Mitov, JHEP 1605 (2016) 034 [arXiv:1601.05375 [hep-ph]].

[6] J. Currie, A. Gehrmann-De Ridder, T. Gehrmann, E. W. N. Glover, A. Huss and J. Pires, Phys. Rev. Lett. 119 (2017) no.15, 152001 doi:10.1103/PhysRevLett.119.152001 [arXiv:1705.10271 [hep-ph]].

[7] J. Currie, A. Gehrmann-De Ridder, T. Gehrmann, E. W. N. Glover, A. Huss and J. Pires, [arXiv:1807.03692 [hep-ph]].

[8] J. Currie, T. Gehrmann and J. Niehues, Phys. Rev. Lett. 117 (2016) no.4, 042001 doi:10.1103/PhysRevLett.117.042001 [arXiv:1606.03991 [hep-ph]].

[9] A. Gehrmann-De Ridder, T. Gehrmann, N. Glover, A. Huss and D. Walker, arXiv:1807.09113 [hep-ph].

[10] M. Grazzini, S. Kallweit and M. Wiesemann, arXiv:1711.06631 [hep-ph].

[11] M. Grazzini, S. Kallweit, D. Rathlev and M. Wiesemann, JHEP 1705 (2017) 139 [arXiv:1703.09065 [hep-ph]].

[12] D. de Florian, M. Grazzini, C. Hanga, S. Kallweit, J. M. Lindert, P. Maierhöfer, J. Mazzitelli and D. Rathlev, JHEP 1609 (2016) 151 [arXiv:1606.09519 [hep-ph]].

[13] R. Boughezal, F. Caola, K. Melnikov, F. Petriello and M. Schulze, Phys. Rev. Lett. 115 (2015) no.8, 082003 [arXiv:1504.07922 [hep-ph]].

[14] J. Gaunt, M. Stahlhofen, F. J. Tackmann and J. R. Walsh, JHEP 1509 (2015) 058 doi:10.1007/JHEP09(2015)058 [arXiv:1505.04794 [hep-ph]].

[15] R. Boughezal, J. M. Campbell, R. K. Ellis, C. Focke, W. T. Giele, X. Liu and F. Petriello, Phys. Rev. Lett. 116 (2016) no.15, 152001 [arXiv:1512.01291 [hep-ph]].

[16] R. Boughezal, J. M. Campbell, R. K. Ellis, C. Focke, W. Giele, X. Liu, F. Petriello and C. Williams, Eur. Phys. J. C 77 (2017) no.1, 7 [arXiv:1605.08011 [hep-ph]].

[17] M. Cacciari, F. A. Dreyer, A. Karlberg, G. P. Salam and G. Zanderighi, Phys. Rev. Lett. 115 (2015) no.8, 082002 [arXiv:1506.02660 [hep-ph]].

[18] Z. Tulipánt, A. Kardos and G. Somogyi, Eur. Phys. J. C 77 (2017) no.11, 749 [arXiv:1708.04093 [hep-ph]]. 
[19] V. Del Duca, C. Duhr, A. Kardos, G. Somogyi and Z. Trócsányi, Phys. Rev. Lett. 117 (2016) no.15, 152004 [arXiv:1603.08927 [hep-ph]].

[20] V. Del Duca, C. Duhr, A. Kardos, G. Somogyi, Z. Szőr, Z. Trócsányi and Z. Tulipánt, Phys. Rev. D 94 (2016) no.7, 074019 [arXiv:1606.03453 [hep-ph]].

[21] F. Caola, K. Melnikov and R. Röntsch, Eur. Phys. J. C 77 (2017) no.4, 248 [arXiv:1702.01352 [hep-ph]].

[22] F. Caola, G. Luisoni, K. Melnikov and R. RŽntsch, Phys. Rev. D 97 (2018) no.7, 074022 doi:10.1103/PhysRevD.97.074022 [arXiv:1712.06954 [hep-ph]].

[23] G. F. R. Sborlini, F. Driencourt-Mangin and G. Rodrigo, JHEP 1610 (2016) 162 [arXiv:1608.01584 [hep-ph]].

[24] F. Herzog, JHEP 1808 (2018) 006 [arXiv:1804.07949 [hep-ph]].

[25] F. A. Dreyer and A. Karlberg, Phys. Rev. Lett. 117 (2016) no.7, 072001 doi:10.1103/PhysRevLett.117.072001 [arXiv:1606.00840 [hep-ph]].

[26] F. Dulat, B. Mistlberger and A. Pelloni, arXiv:1710.03016 [hep-ph].

[27] J. Currie, T. Gehrmann, E. W. N. Glover, A. Huss, J. Niehues and A. Vogt, JHEP 1805 (2018) 209 doi:10.1007/JHEP05(2018)209 [arXiv:1803.09973 [hep-ph]].

[28] L. Magnea, E. Maina, G. Pelliccioli, C. Signorile-Signorile, P. Torrielli and S. Uccirati, arXiv:1806.09570 [hep-ph].

[29] L. Magnea, E. Maina, P. Torrielli and S. Uccirati, PoS RADCOR 2017 (2018) 035 doi:10.22323/1.290.0035 [arXiv:1801.06458 [hep-ph]].

[30] L. Magnea, E. Maina, P. Torrielli and S. Uccirati, PoS RADCOR 2017 (2018) 043 doi:10.22323/1.290.0043 [arXiv:1801.06462 [hep-ph]].

[31] L. Magnea, E. Maina, G. Pelliccioli, C. Signorile-Signorile, P. Torrielli and S. Uccirati, in preparation.

[32] C. Duhr and T. Gehrmann, Phys. Lett. B 727 (2013) 452 [arXiv:1309.4393 [hep-ph]].

[33] Y. Li and H. X. Zhu, JHEP 1311 (2013) 080 [arXiv:1309.4391 [hep-ph]].

[34] L. Magnea and G. F. Sterman, Phys. Rev. D 42 (1990) 4222.

[35] S. Catani, Phys. Lett. B 427 (1998) 161 [hep-ph/9802439].

[36] G. F. Sterman and M. E. Tejeda-Yeomans, Phys. Lett. B 552 (2003) 48 [hep-ph/0210130].

[37] L. J. Dixon, L. Magnea and G. F. Sterman, JHEP 0808 (2008) 022 [arXiv:0805.3515 [hep-ph]].

[38] E. Gardi and L. Magnea, JHEP 0903 (2009) 079 [arXiv:0901.1091 [hep-ph]].

[39] E. Gardi and L. Magnea, Nuovo Cim. C 32N5-6 (2009) 137 [Frascati Phys. Ser. 50 (2010)] [arXiv:0908.3273 [hep-ph]].

[40] T. Becher and M. Neubert, Phys. Rev. Lett. 102 (2009) 162001 Erratum: [Phys. Rev. Lett. 111 (2013) no.19, 199905] [arXiv:0901.0722 [hep-ph]].

[41] T. Becher and M. Neubert, JHEP 0906 (2009) 081 Erratum: [JHEP 1311 (2013) 024] [arXiv:0903.1126 [hep-ph]].

[42] I. Feige and M. D. Schwartz, Phys. Rev. D 90 (2014) no.10, 105020 [arXiv:1403.6472 [hep-ph]]. 
[43] Ø. Almelid, C. Duhr and E. Gardi, Phys. Rev. Lett. 117 (2016) no.17, 172002 [arXiv:1507.00047 [hep-ph]].

[44] Ø. Almelid, C. Duhr, E. Gardi, A. McLeod and C. D. White, JHEP 1709 (2017) 073 doi:10.1007/JHEP09(2017)073 [arXiv:1706.10162 [hep-ph]].

[45] D. A. Kosower, Nucl. Phys. B 552 (1999) 319 [hep-ph/9901201].

[46] S. Catani and M. Grazzini, Phys. Lett. B 446 (1999) 143 [hep-ph/9810389].

[47] S. Catani and M. Grazzini, Nucl. Phys. B 570 (2000) 287 [hep-ph/9908523].

[48] J. M. Campbell and E. W. N. Glover, Nucl. Phys. B 527 (1998) 264 [hep-ph/9710255].

[49] Z. Bern, V. Del Duca, W. B. Kilgore and C. R. Schmidt, Phys. Rev. D 60 (1999) 116001 [hep-ph/9903516].

[50] S. Catani and M. Grazzini, Nucl. Phys. B 591 (2000) 435 [hep-ph/0007142]. 